\title{
Leaf-structure patterning for antireflective and self-cleaning surfaces on Si-based solar cells
}

\author{
Zhongjia Huang ${ }^{\mathrm{a}, b^{*}}$, Congcong Cai ${ }^{\mathrm{a}}$, Long Kuai ${ }^{\mathrm{c}}$, Taohai $\mathrm{Li}^{\mathrm{d}}$, Marko Huttula ${ }^{\mathrm{b}}$, and Wei Cao ${ }^{\mathrm{b}^{*}}$
}

a School of Mechanical and Automotive Engineering, Anhui Polytechnic University, Wuhu 241000, China

b Nano and Molecular Systems Research Unit, University of Oulu, P.O. Box 3000, FIN-90014 Oulu, Finland

${ }^{\mathrm{c}}$ College of Biological and Chemical Engineering, Anhui Polytechnic University, Wuhu 241000, China

d College of Chemistry, Key Lab of Environment Friendly Chemistry and Application in Ministry of Education, Xiangtan University, Xiangtan, China

*Corresponding authors

E-mail addresses: hzj@ahpu.edu.cn (Z.Huang), wei.cao@oulu.fi (W.Cao)

Abstract: As the naturally evolved sunlight harvester, plant foliage is gifted with dedicated air-leaf interfaces countering light reflections and ambient ruins, yet offering antireflective and selfcleaning prototypes for manmade photovoltaics. In this work, we report on an ecological and bioinspired coating strategy by replicating leaf structures onto $\mathrm{Si}$-based solar cells. Transparent photopolymer with leaf surface morphologies was tightly cured on Si slabs through a facile double transfer process. After bio-mimicked layer coverages, sunlight reflection drops substantially from more than $35 \%$ down to less than $20 \%$ once lotus leaf was employed as the master. Consequentially, $10.9 \%$ gain of the maximum powers of the photovoltaic is obtained. The leaf replicas inherited their masters' hydrophobicity which is resistant to acidic and basic conditions. Physically adhered dusts are easily removed by water rolling. Lightwave guidance mechanism among air-polymer-Si interfaces is explicated through optical simulations, while wettability through the morphological impacts on hydrophobic states. Taking advantages of varieties of foliage species and surface 
structures, the work is hoped to boost large-scale industrial designs and realizations of the bionic antireflective and superhydrophobic coating on future solar cells.

Keywords: Biomimetic, sunlight harvesting, antireflection, hydrophobicity

\section{Graphical abstract}

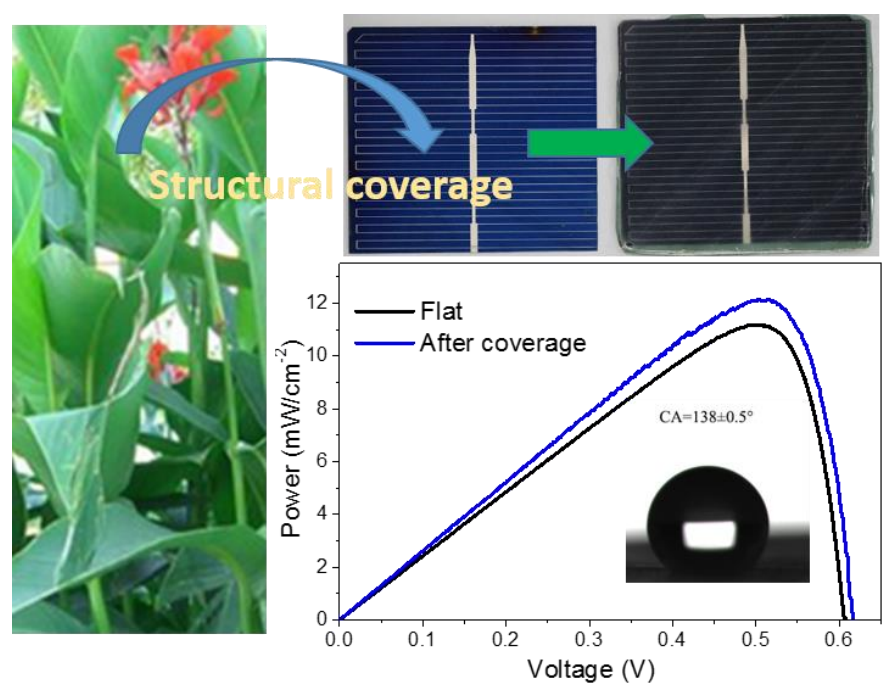




\section{Introduction}

Light exhibits reflection when it impinges on interface between two media with different refractive indices. Such a reflection rate of sunlight reaches more than $35 \%$ on the surface of polished silicon, the dominant material to harvest sunlight in photovoltaic (PV) modules. Bare silicon slab is physically brittle and easily oxidized with time. The physical property poses survivals of modules in different ambiences, whilst the latter leads to surface recombination loss in the cells and decrease of PV efficiency (Hirst and Ekins-Daukes, 2011). In most cases, PV modules are exposed to various natural weather conditions. They are easily covered by dusts and organic wastes from plant, animal, and industrial pollutants (Said et al. 2015). The unwanted light reflection and dirt ask for proper shielding materials onto the bare Si slabs. For practical applications, such materials should be antireflective (AR), transparent, protective, durable and self-cleaning. Furthermore, the material synthesis route should be easy and cheap, featuring in large-scale industrial fabrication.

In a typical Si-PV module, the silicon slab is folded by a glass shield above an encapsulation layer of ethyl vinyl acetate (EVA). Patterning the glass will not only need additional treatments to the chemically inert glass itself, but also ask for a clear clue in selecting patterns from tremendous micro- and submicro-types. Another route is to directly engineer the Si surface with AR structures, such as through colloidal lithography (Dev et al. 2014), wet-etching processes (Adamian et al. 2000), ion etching (Bien et al. 2012), etc. Various micro- and submicro- structures have been used in PV cells (Zang et al. 2013), or on the Si slabs too (Bichotte et al. 2017; Jovanov et al. 2017). Albeit successes in antireflection designs, surface structure durability and wettability remain unclear within these treatments. Additionally, the costs and limits of pattern types bar practical applications in large scales.

When talking about solar energy conversions, the primary function of plant foliage shall never be neglected. Photosynthesis on leaves supplies both oxygen and organic matters on earth. After 
millions of years of natural evolution, surface morphologies are not only benefiting solar radiance harvesting, but also apt to protections and environmental adaptations. The upper epidermis "shields" of the natural sunlight harvesting systems play a similar but crucial role to these of the ideal covers on manmade PV systems. The rich plant species further provide a large library for the coating types. Recently, these bio-inspired structures have been proved efficiently enhancing solar energy conversions on PV cells, thanks to their lightwave guidance nature (Huang et al. 2015, 2016). However, the bio-structures are mainly patterned onto soft species such as transparent polymers which are further folded onto possible active units (Chen et al. 2015). Later, direct replicating biostructures on to PV unit was also reported, but the functional sizes of the surfaces were very much limited due to the tiny leaf masters (Vüllers et al. 2016). Besides, physical mechanisms of the antireflective property and hydrophobicity were not revealed. A facile, precise and reliable route to replicate the bioinspired structures directly onto large inorganic Si slab is required, together with understanding of physical origins beyond multi-functionalities of the structures.

In this paper, we report on replicating leaf surface structures directly onto bare silicon surfaces to form antireflective and self-cleaning coatings through a facile double transfer method. Light reflection on the patterned surface is suppressed lower around $20 \%$ determined via UV-Visible spectroscopy. Consequentially, a gain of more than $10 \%$ for the maximum power was reached on the patterned silicon photovoltaics. Benefiting from the AR and wettability of the master leaves and physical and chemical advances of the cured photopolymer, a maximum value of $150( \pm 0.5)^{\circ}$ is found for the static water contact angle (CA) from the Lotus replica, and $20( \pm 0.5)^{\circ}$ for the rolling angle from the same replicated surface. Thanks to morphological advances, hydrophobicity is resistant to ambient liquid $\mathrm{pH}$ values. Lightwave guidance mechanism was clarified through optical simulations, and wettability through hydrophobic state analysis. Such an ecological and bio-inspired method paves a route for large-industrial scale fabrications of the reliable coatings with antireflective, transparent and self-cleaning properties. 


\section{Experimental methods}

\subsection{Master leaf selection}

The strategy debuts from carefully observations of natural leaves and their responses to ecological systems. Here, fresh master leaves were collected from the following 4 plants: lotus (Nelumbo nucifera), Indian shot (Canna indica), grape (Vitis vinifera L.) and bamboo (Phyllostachys edulis). Lotus leaf is famous for its superhydrophobic property. The structures leading to the property has been a research focus since the beginning of the century (Sun et al. 2005). It's worth mentioning that such water repelling nature does not exclusively belong to aquatic plants. The Indian shot, a terrestrial plant from the Cannaceae family, is also superhydrophobic (Sharma and Mattaparthi, 2014), yet distinguishing themselves in tropic homegarden systems(Gajaseni, 1999). As a typical liana plant, the grape possesses high solar energy harvesting efficiency in the photosynthesis for fruit production. We further notice that bamboo grows in warm moist environment, but the leaf surface feels coarser than many other tall wooden plants. This perennial evergreen plant features fast growth and prompt regeneration capacity, referring to very good light harvesting features. Furthermore, the leaf extract/main components are used as efficient inhibitors against stainless steel corrosions (Li et al. 2014).

\subsection{Double transfer process}

A schematic of fabrication steps for the coating is shown in Fig. 1. To start, fresh leaf masters were firstly cleaned with ethanol, and then dried with drier blow. Leaf types are selected to these with good hydrophobic properties, and with fast growth speed. They were then stuck in the die as shown in Fig. 1(b). Fig 1 (c) shows a mould with negative leaf structure was copied to the polydimethylsiloxane (PDMS). This undergoes the same procedure as described in our previous work (Huang et al. 2015). A negative master of PDMS was formed (Fig. 1(d)) and ready for photopolymer curing. Here, the Norland Optical Adhesive 63 (NOA63) photopolymer was 
employed as the coverage materials on polished bare silicon slab or Si PV cells without any coverage. Besides its feasibility in curing, the polymer also advances in stability in hierarchical structure moulding (Leem et al. 2016). The Si slabs were cut to as $1 \times 1 \mathrm{~cm}^{2}$ to fit active regions of the plant leaves. The PV cells have a dimension of $5 \times 5 \mathrm{~cm}^{2}$, nominal filling factor of 0.75 , opencircuit voltages of $0.6 \mathrm{~V}$ and short-circuit current density of $24 \mathrm{~mA} / \mathrm{cm}^{2}$. In Fig. 1(e), the NOA63 liquid was evenly placed on the negative PDMS mould. The amount of NOA63 is enough to cover PDMS, and slight above the negative edges. Later, the silicon upper surface facing to the light was very gently placed to the photopolymer. Then, NOA63 was cured under UV radiation for 6-8 minutes until its solidification. The negative mould can be mechanically torn and detached from the PV surfaces, leaving the polymer with positive leaf structures ready on the Si surfaces as Fig. 1(f) shows.

(a)
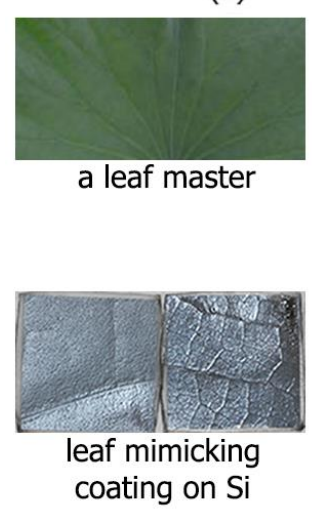

(f) (b)

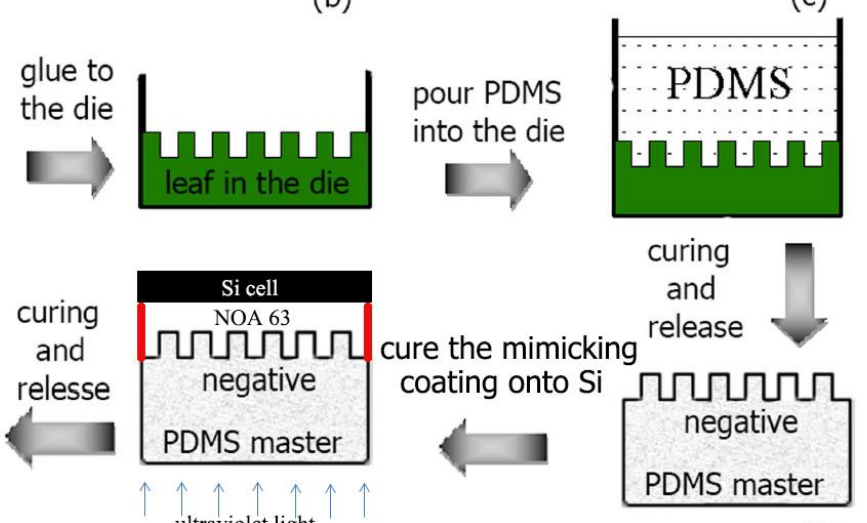

(e)

(d)

Fig. 1 Schematic of fabrication steps (a) A fresh leaf was taken as a master module (b) The leaf was stuck in the die (c) Polydimethylsiloxane was deposited on the leaf to get negative structures of the master. (d) A flexible negative mould was covered with an anti-sticking layer (e) Viscous NOA63 was poured onto the negative mould and Si slab or bare cell was covered onto NOA63. The NOA63 was then polymerized using Ultraviolet light. (f) After polymerization, the detached NOA63 mimicked the master leaf surface structures on Si slab.

\subsection{Characterizations}


Morphological determinations of the replicated photopolymers were carried out on a Hitachi S-4800 scanning electron microscope (SEM). For comparison purposes, corresponding master leaves were measured through the SEM too. Leaf samples were coated with carbon to increase conductivity during the measurement. All photos, except the one from real plant, in the present work were shot under fluorescence lighting with a Cannon digital camera without any correction through picture software. Optical properties of the coated surfaces were measured through a UVVisible/NIR Spectrophotometer UH4150. The surface reflection and absorption curves were measured at a wavelength region from 300-800 nm, covering all visible light region of the solar spectrum. Analysis of the wettability was performed through a contact angle apparatus JY-82A from Chengde Dingsheng Testing Machine. Co. Ltd. By adding $\mathrm{HCl}$ or $\mathrm{NaOH}$ into water, the liquid was adjusted acidic or basic, and $\mathrm{CA}$ variations were measured at a $\mathrm{pH}$ range of 4-10. In the photovoltaic measurement, a solar simulator (SAN-EI ELECTRIC XES-40S1) under AM 1.5G illumination at $100 \mathrm{~mW} / \mathrm{cm}^{2}$ power density output was employed to obtain the $\mathrm{I}-\mathrm{V}$ curves from silicon PV cells without and with the leaf structure coverages.

\section{Simulations}

To understand the physical mechanisms leading to AR nature, optical simulations were carried on the $\mathrm{SiO}_{2}$ surface structures with the $0.05 \mu \mathrm{m} \mathrm{Si}$ substrates through the software of finitedifference-time-domain (FDTD) solutions $^{\mathrm{TM}}$. The simulation time was set to 10000 fs. A plane wave was selected as a simulated light source, and light wavelength covered range from $300 \mathrm{~nm}$ to $800 \mathrm{~nm}$. The $\mathrm{SiO}_{2}$ is employed here due to its wide application in PV cells, and similar refractive index to the one of NOA 63 above $380 \mathrm{~nm}$. Its thickness is set to $15 \mathrm{um}$, and refractive index of 1.55 , very close to the value of 1.56 from NOA 63 . Boundary condition are set as 'periodic' in $\mathrm{X}$ and $\mathrm{Y}$ axis direction, and 'perfectly matched layer' in $\mathrm{Z}$ axis direction. During the simulation, lightwave guidance mechanism among the interfaces was conducted by using an abstracted bamboo structure as the model. Based on the microscopic determinations, it is constructed by one micro- 
cone surrounded by four microspheres (see later in the text). Detailed computational methodology can be found e.g., in (Huang et al. 2016).

\section{Results and discussion}

\subsection{Morphology}

The SEM images of the master leaf surfaces are shown in Fig. 2 (a)-(d), with the corresponding replicas in Fig. 2 (e)-(h). Hereafter, we name the replica as PPR@PLN, where PPR refers to the photopolymer replica and PLN to the Plant Name. In general, the replica surface morphologies are almost identical to these of their masters, denoting the success of the direct transfer process.

Surface morphologies of the replicas vary from plant families. Lotus leaf has micrometer papillae where additional nanorods are distributed on them. This structure has been thoroughly studied elsewhere due to its effects in wettability (Koch and Barthlott, 2009). The Indian shot has rectangles separated by concaved slots. These rectangles have varied shapes ranging from squares to long rectangles within tens of micrometer. Typical plant nanorods are also distributed on the rectangles too (see Fig.2(j)). Similar to water repelling structure on lotus leaf, micro-cones also widely exist on the bamboo leaf, as shown in Fig. 2 (c) and (g). However, the distribution is less intensive than the one on lotus leaf, yielding differences in interfacial impacts to ambient conditions. As denoted in SEM images, bamboo leaf has long fibers orienting parallel to the main vein. On grape leaf, microbubbles with $\sim 30 \mu \mathrm{m}$ diameter were additionally folded by vein chains as shown in Fig. 2 (h). However, these chains are less visible in the replica, due to their rather loose attachment on the surface and detachment during the double transfer process.

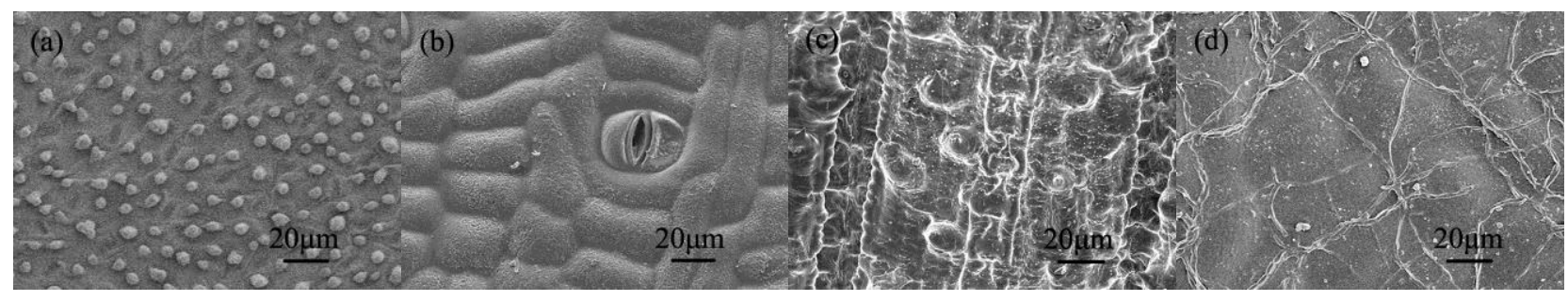



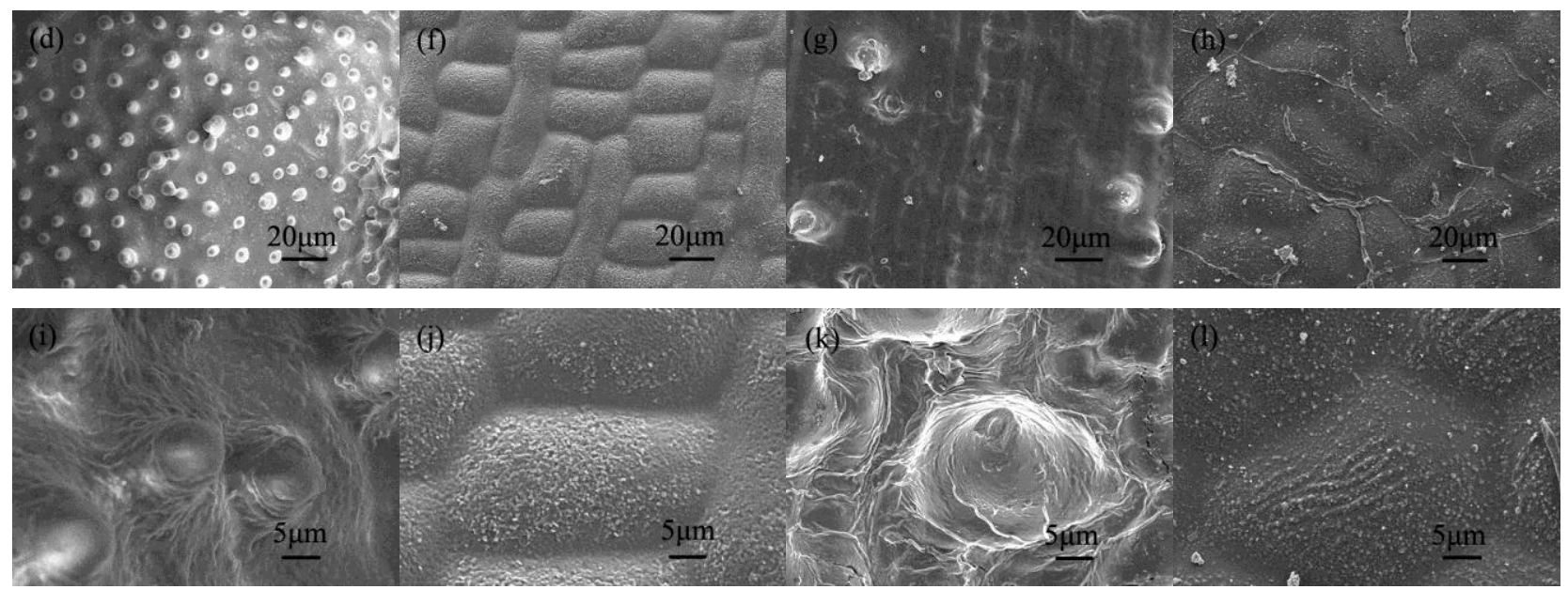

Fig. 2. Scanning electron microscopic (SEM) determination of surface morphologies of photopolymer replicas compared with these of their master leaves. (a)-(d), SEM images master leaf structures from Lotus, Indian shot, Bamboo, Grape, (e)-(h), from their corresponding replicas, and (i)-(1) the magnified SEM images of the replica.

To test durability of the replica, four heating-cooling cycles were performed for the PPRs coated Si slabs. Figure 3 shows the SEM images of surface morphologies after the heating-cooling cycles. Structure are stable under $80{ }^{\circ} \mathrm{C}$ for more than 32 hours heating, and 64 hours cooling. No piling off or bubbling was found in the films. Consider the work temperature of the solar panels, the temperature applied here is extremely harsh.

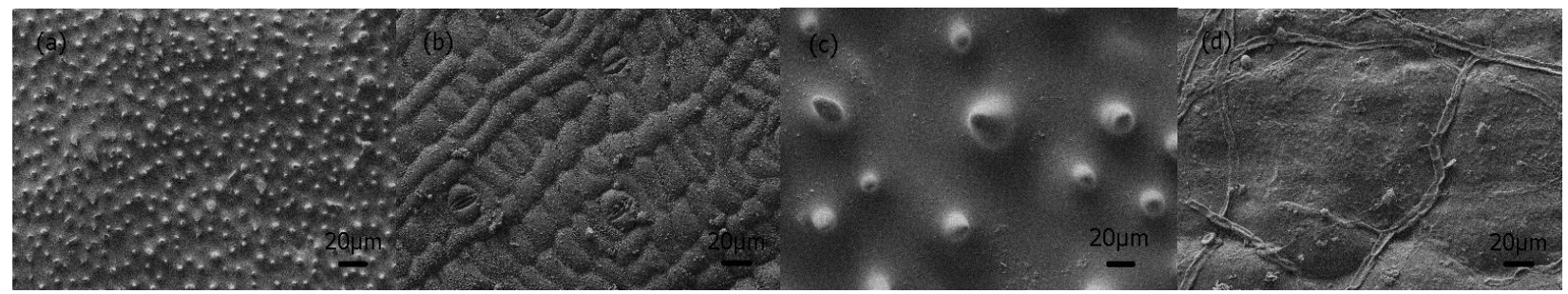

Fig. 3 SEM images of surface morphologies of heating-cooling cycles test samples of mimicked the master leaf surface structures from (a) Lotus (b) Indian shot (c) Bamboo (d) Grape.

The photopolymer is firmly cured on inorganic Si slab surface. This can be seen in cross sectional SEM in Figure 4 (a) where the clear organic-inorganic interface is formed with a gluing section around $20 \mu \mathrm{m}$, with leaf structures on the surface. The cured polymer is also tightly cured 
with no obvious air bubbles inside of the film (right side of Fig. 4(a)). Clearly, the degassing process was successfully carried out during the coating procedure. The top layer of leaf-mimicking polymers was also investigated by the cross sectional SEM. As shown in Fig. 4(b), the lotus papillae have a height around $10 \mu \mathrm{m}$ above the uneven surface of micrometer scale roughness.
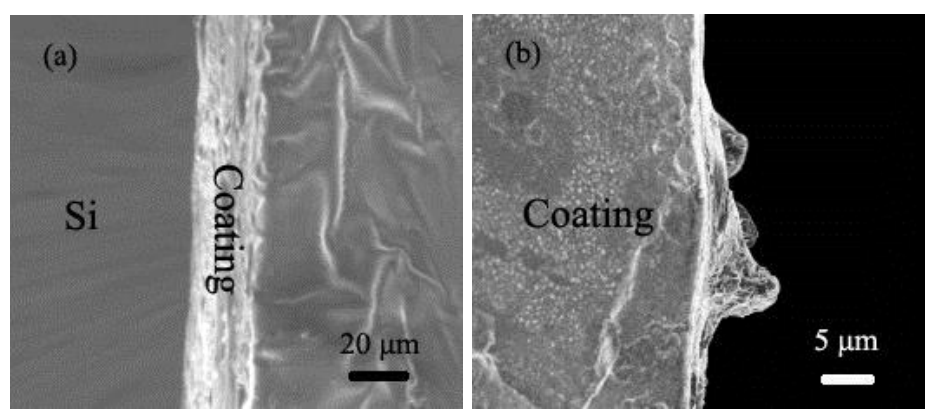

Fig. 4. Cross-sectional scanning electron microscopies of photopolymer-Si interface (a), and individual papillae of the lotus leaf replica curved on flat Si slab (b).

\subsection{Optical property}

Imprinting microstructures of leaves will decrease surface light reflection of the polymersilicon system. To visualize the AR fact of present work strategy, we photographed PPR@PLN together with the bare silicon, as shown in Fig. 5. The metallic greyish color of silicon disappeared after coating $\mathrm{Si}$ with the leaf-mimicking structures. The intrinsic shininess is muted, decreasing surface light reflection. The flat Si surface is also modified to the coarse ones of leaf structures. This can be seen in Fig. 5 that whilst the PPR@ Indian shot is flat due to selection of a smooth area of the large master leaf, other three PPRs show structures of the master leaves with clear veins and textures on them. The patterned surfaces substantially decrease unwanted light reflection as viewed in the figure where the image of the card on mirror like bare Si surface disappeared on the PPRs. 


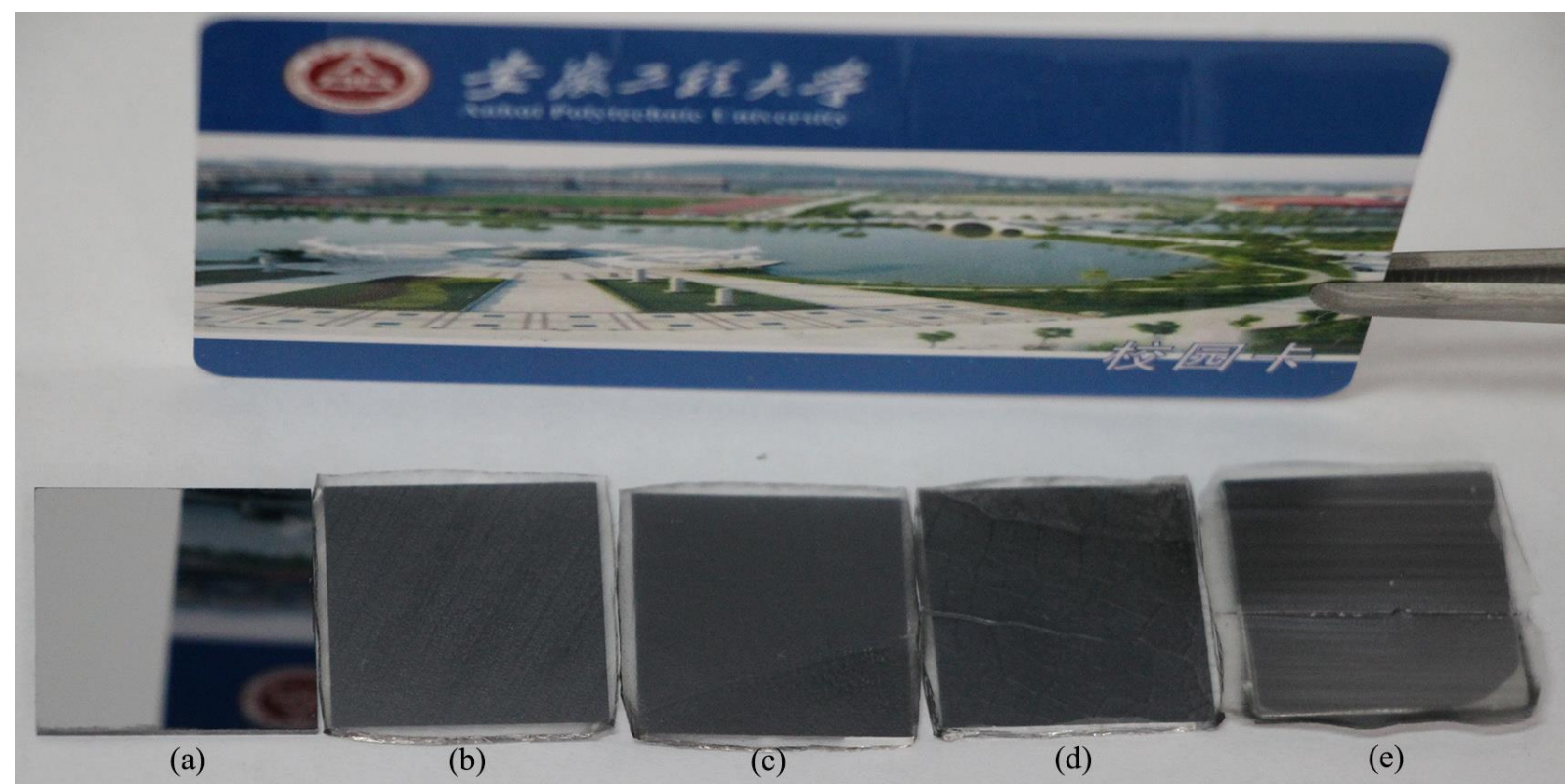

Fig. 5. Photo of polished silicon coated with leaf replicas with their masters of (a) bare polished silicon, (b) PPR@Indian shot, (c) PPR@Lotus, (d) PPR@Grape and (e) PPR@Bamboo from right to left. The very left is a bare polished silicon slab for comparison purpose.

Quantitative determinations of light reflection and absorption were also carried out by using radiation from a solar simulator as the incident source. The light reflectance (in percent) is depicted in Fig. 6(a) for the wavelength ranging from $300 \mathrm{~nm}$ to $800 \mathrm{~nm}$, and absorption in 6(b) in the same range. At wavelengths below $\sim 380 \mathrm{~nm}$ some of light absorption is attributed to the absorption of NOA 63 polymer (Choi et al. 2015). At longer wavelength, the intrinsic absorption from the polymer is negligible according to the datasheet of the polymer. The reflectance from the silicon surface decreases drastically from more than $35 \%$ down to $\sim 20 \%$. Such an effect is very close to the AR design by using dedicated $\mathrm{TiO}_{2}$-Graphene strategy on the polished Si surface (Shi et al. 2013). All reflectance decreases smoothly in the visible range, as a result of light-matter interaction. The PPR@Lotus possesses the smallest light reflectance, followed by PPR@Indian shot, PPR@Bamboo, and PPR@grape. Consequently, biomimicking coating substantially increases light absorptions in the silicon slabs at wavelengths $380 \mathrm{~nm} \sim 800 \mathrm{~nm}$ as shown in Fig. 6(b). In general, the trend of absorption also agrees with the one of reflection, with PPR@Lotus topping. Slight variation was 
found for the absorption rates of PPR@Bamboo and PPR@Indian shot compared the reflection data. This is due to the differences of light absorptions within the transparent photopolymers of different thicknesses. It is worth noting that the present AR coating cannot reach reflectance to the low values as yielded from the heterojunctional interfacial engineering (Leem et al. 2016; Zhu et al. 2013). The intrinsic refractive index of transparent photopolymer is still very different from these of the silicon and air. However, compared with multilayer AR coatings, the present work is straightforward and easily reached on large surfaces.
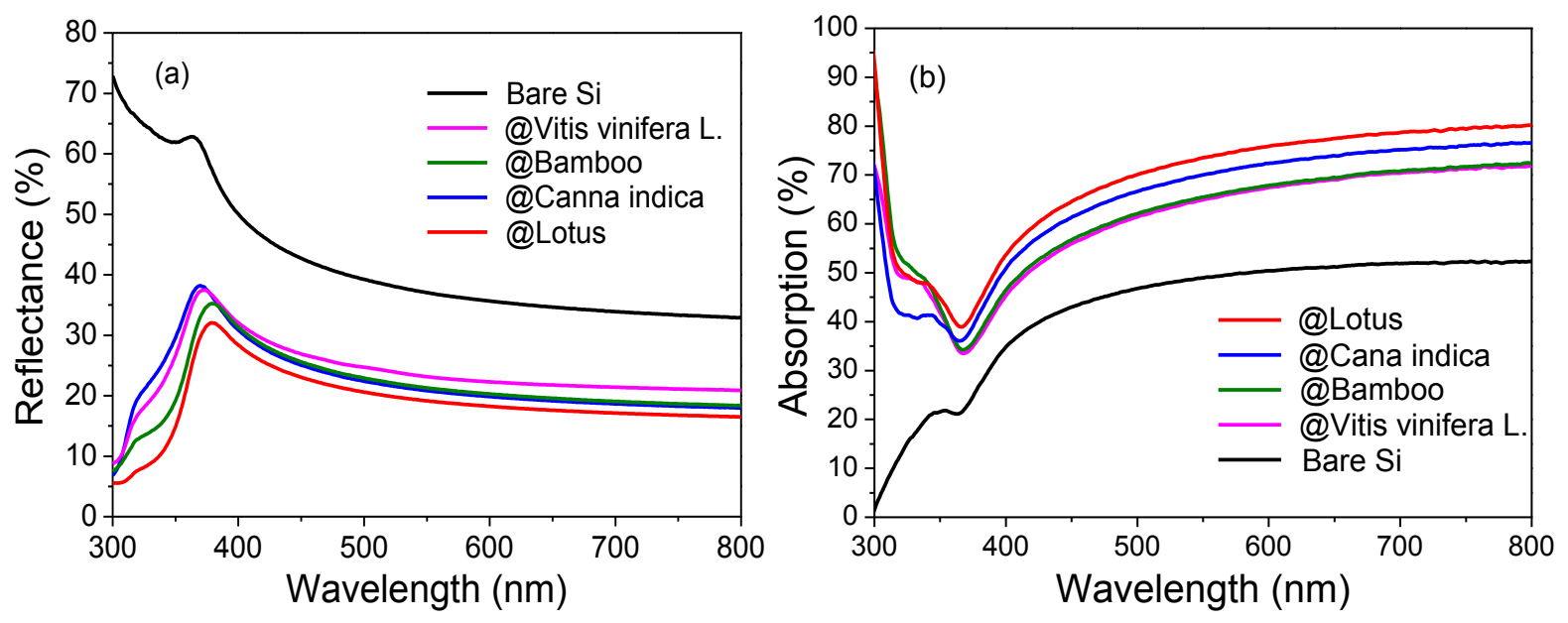

Fig. 6. Reflectance (a) and absorption (b) of plant photopolymer coated silicon surfaces determined through UV-visible spectroscopy.

An optical simulation on bamboo structures was employed to interpret the lightwave guidance among different media. Based on morphological determinations as shown in Fig.2, the abstracted model in Fig. 7 (a) was constructed with rounded tip mircocones distributed on sub-millimetre fibres. The computed reflection rate curve was shown in Fig. 7(b), with the animation in Supplementary Video 1. Reflection curve from pure Si was also calculated for comparison purposes. From the video, it is clear that the lightwave is scattered, and diffused inside of the transparent polymer. At the polymer-Si interfaces, the diffused light scattered back, but not able to be reflected above the polymer-air interfaces. Such a diffusion and trapping of the lightwaves lead to a drastic decrease from $35 \%$ on Si down to $20 \%$ on the module, as shown in Fig. 7 (b). Additionally, the 
calculated results agree well with the experimental ones (see. Fig. 6 (a)). Inconsistences were found e.g. at the lower wavelength region $<400 \mathrm{~nm}$ for both bare Si and coated surfaces. This is mainly due to differences of intrinsic properties of materials employed in experiments and computations. The thin slab applied in the simulation allows more light transmissions above the Si absorption threshold energy, leading to lower backward reflection (Nowak et al. 2014). As for the coated surfaces, it is noticed a large absorption below $400 \mathrm{~nm}$ happens on the NOA 63 photopolymer surface, but not so on the $\mathrm{SiO}_{2}$ one in the simulation model. This is mainly because NOA 63 polymer owns different absorption coefficient than $\mathrm{SiO}_{2}$ at lower wavelengths below $380 \mathrm{~nm}$ (Choi et al. 2015). Nevertheless, solar spectrum has rather small abundances at this wavelength region. Thus, differences in theoretical and experimental models are not crucial to light harvesting, while the glass coverage may be more practical for future bio-inspired coating on PV cells eventually. It is also noticed that external quantum efficiency (EQE) of the solar cells is affected by many factors such as coverages, electrodes, grade of Si, sealing technics, etc. It's a systematic and dedicated project to compare internal and external factors influencing QE, yet, out of the main scope of the present work. Alternatively, we evaluated the optic loss by comparing the reflectance from $\mathrm{SiO}_{2}$ coverages with and without leaf structure patterning. Apparently, the reflectance with the leaf structure pattern is much lower than the one with only flat $15 \mu \mathrm{m} \mathrm{SiO}{ }_{2}$ coverage (blue curve in Fig. 7(b)). The oscillation mainly arises from the light interference of front and back scattering of the incident beam. Here, by using bio-inspired structures, our result has shown substantial decrease of the light reflection. As a result, the EQE of the solar cell can be decreased accordingly. 

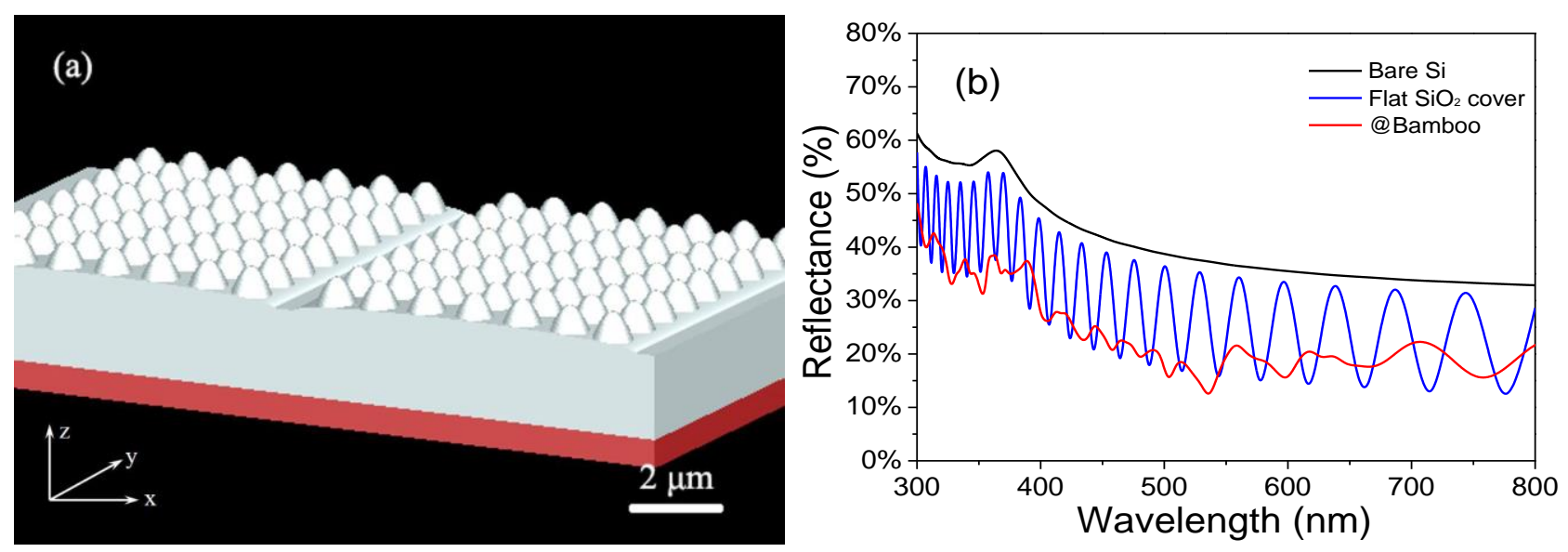

Fig. 7. Micro morphology of abstracted PPR@bamboo model (a) for optical simulation, and calculated reflectance curves from the model, flat $\mathrm{SiO}_{2}$ cover and bare silicon surfaces (b).

It has been noticed some light scattering nature also exist in SV-1. To clarify the computational finding, we further carried out light scattering experiment. A self-stand PPR@bamboo was formed with the same photopolymer, following the preparation route given by (Huang et al. 2015). The scattering test was under the incident shining of a red color laser with a wavelength $650 \mathrm{~nm}$ and a beam diameter $3 \mathrm{~mm}$. Laser beam passed through the PPR@Bamboo and illuminated large areas. The scattered beams were photographed and shown in Fig. 8 (a) and (b). In contrast to a $4 \mathrm{~mm}$ spot after laser passing through a smooth NOA 63 and travelling $200 \mathrm{~mm}$, the illuminated areas are rather large due to forward scatterings of the laser.

(a)

(b) 
Fig. 8. Light scattering spots. (a) shows the light scattering effect of PPR@Bamboo when a laser with a diameter of $3 \mathrm{~mm}$ passing though the mimicked bamboo leaf structure NOA63 polymer. (b) shows the light scattering effect of smooth NOA 63 polymer.

\subsection{Gains on solar cells}

Coating bio-mimicked leaf structures onto exist PV panels will yield increase of light harvesting efficiency. Images of the bare and PPR@ Indian shot cells are shown in Fig.9 (a). Similar to the above results, surface shininess is obviously different after AR coating. In Fig. 9(b), we show the current-voltage curves measured through the standard test on a solar simulator. PV cells coated with leaf structures promotes both open-circuit voltages (OV) and short-circuit currents, while still keeping the similar filling factor of the cells. The voltages measured from the coated cells are larger than the nominal OV value of $0.608 \mathrm{eV}$. PPR@Lotus again yields the best performance to increase the PV gains, followed by the cells covered with Indian shot, bamboo, and grape replicas. The trend of lotus - Indian shot - bamboo - grape is in line with the optic data as shown in Fig.6. A maximum power gain of $10.9 \%$ was found after folding PPR@Lotus onto the bare silicon PV cell as shown in Fig. 9(b). The number turns to $8.6 \%$ (Indian shot), 6.0\% (bamboo) and 5.0\% (grape), for the rest three species respectively. It should be noted here that standard test results are different from these under weak light incident or on photovoltaics with low filling factors in aspects of incident angle, radiance spectral distribution, and internal loss of the cells. The standard test was carried out under 'ideal conditions' with simulated sunlight spectrum, perpendicular incident, parallel light, and room temperature on A-class PV cells. However, in reality, the harvesting efficiency of the PVs is substantially limited by sunlight scattering and absorption (e.g. by smog, cloud, and aerosol), incident angles, temperature, as well as coverages of unwanted moistures or dusts. The class of PV cell is also another factor. Typically, the used or low-class PVs suffer from increases of internal series resistance [Kunz, G. \& Wagner, A. Internal Series Resistance Determination of Only IVCurve under Illumination. 19th Eur. Photovolt. Solar Energ. Conf., 5BV.2.70 (2004).], and extrinsic 
defects [1. Aberle, A. G. Surface Passivation of Crystalline Silicon Solar Cells: A Review. Prog. Photovolt: Res. Appl. 8, 473-487 (2000).]. All of these will lead to differences of the gains for the ideal tests and practical ones.
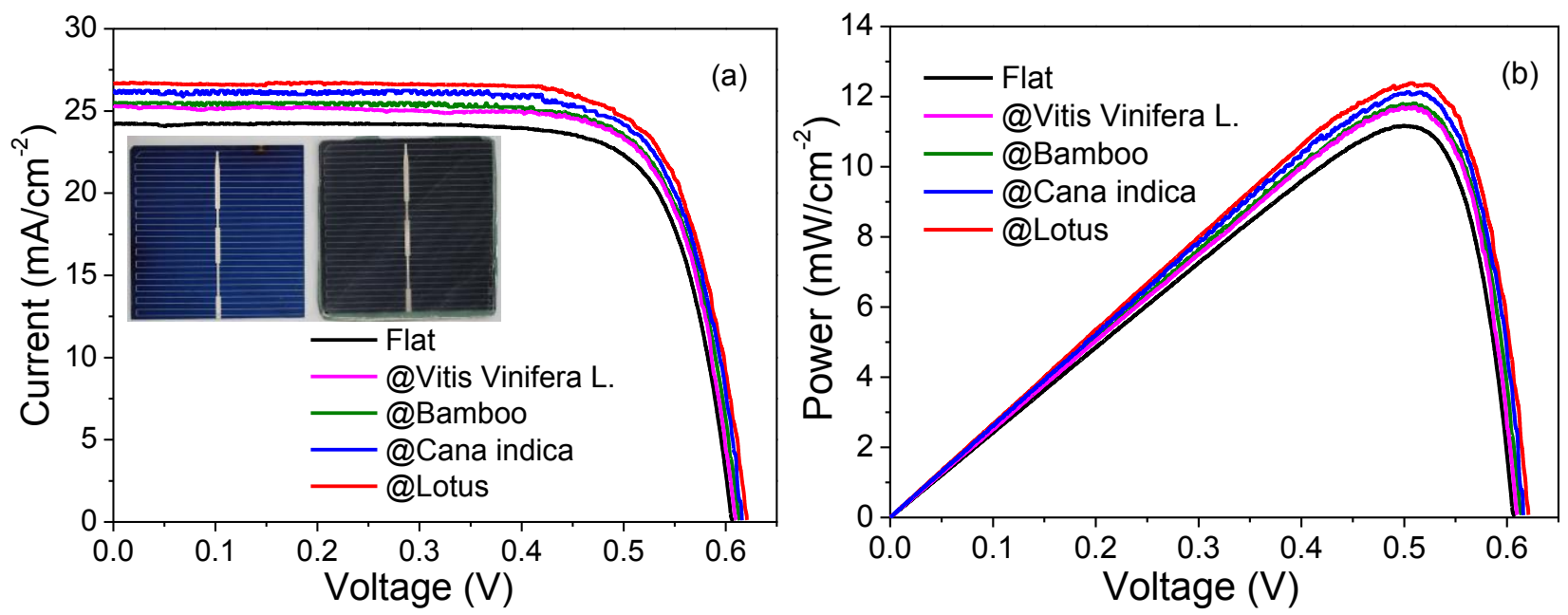

Fig. 9. Current-voltage curves of the silicon photovoltaics covered by different leaf replicas compared with the cell without any coverage measured under solar simulator. Inset of (a) shows a photo of bare silicon photovoltaics (left) and one covered by Indian shot replica (right), while (b) denotes maximum powers of the photovoltaics.

\subsection{Wettability and self-cleaning property}

Inherited from their master leaves, some of the patterned surfaces also possess hydrophobic properties to repel water. Figure 10 gives the CA results measured on top of the biomimicking surfaces without modification of any low surface energy components such as PFOTS. All these CAs are substantially larger than $\sim 90^{\circ}$ of the polished silicon covered by the native oxide layer (Williams et al. 1974). PPR@Lotus is superhydrophobic with a CA value of $150( \pm 0.5)^{\circ}$, in according to the CAs of the lotus leaf, the nanocasted replica (Sun et al. 2005) and the biomimetic counterparts (Guan et al. 2015). The PPR@Indian shot also shows hydrophobicity with a CA of $138( \pm 0.5)^{\mathrm{o}}$ as shown in Fig. $10(\mathrm{~b})$. The CA number decreases to $125( \pm 0.5)^{\mathrm{o}}$ on bamboo replica and $122( \pm 0.5)^{\mathrm{o}}$ on the one of grape (Fig.10(c), (d)). The special wettability originates from the 
roughness nanocasted from the master leaves. Benefiting from the specific roughness of Indian shot and lotus leaves, low air liquid-surface ratio will reduce the contact area of the droplets, forming the Cassie's (Lotus) states (Zhu et al. 2017; Meng et al. 2016) and leading to the present hydrophobicity.

The unique wettability of the replicas results in a small rolling angle. As shown in the snap shots in Fig. 10(e-h), when the biomimicked surface of lotus was tilted by $20( \pm 0.5)^{\mathrm{o}}$, a droplet of 5 $\mathrm{uL}$ started to roll off from the surface. The hydrophobic surface and low rolling angle will gift selfcleaning properties to the PPR coated PV cells. The superhydrophobic surfaces normally own five possible states: Wenzel's state, Cassie's state, the "lotus" state, the transitional state between Wenzel's and Cassie's states, and the "gecko" state. Taking the present surface morphology and water rolling property into consideration, the state of PPRs cured from the photopolymer is preferably the "lotus" state, as a distinct case of the Cassie's state (Zhu et al. 2017).

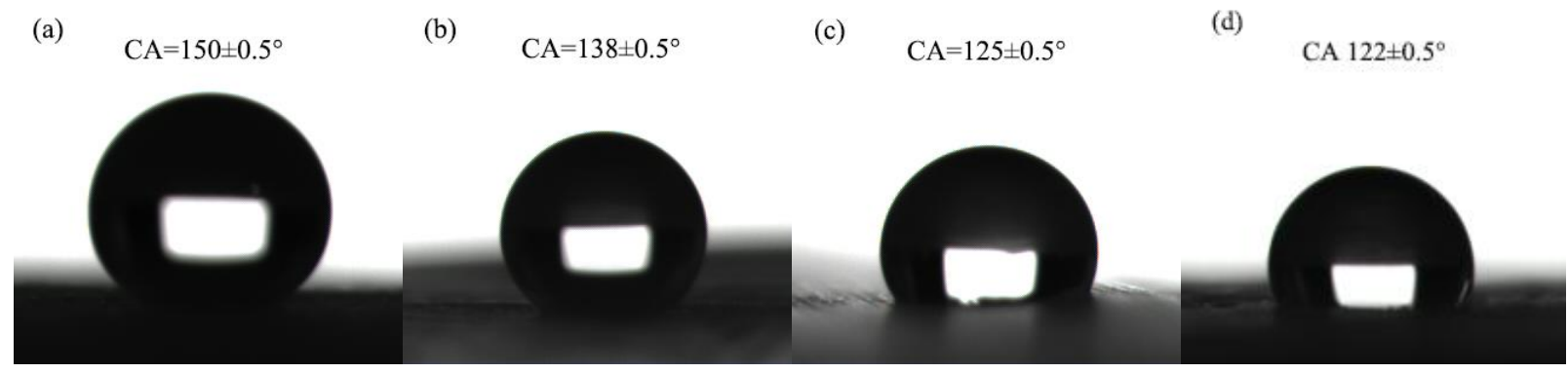

(e)

(f)

(g)

(h)

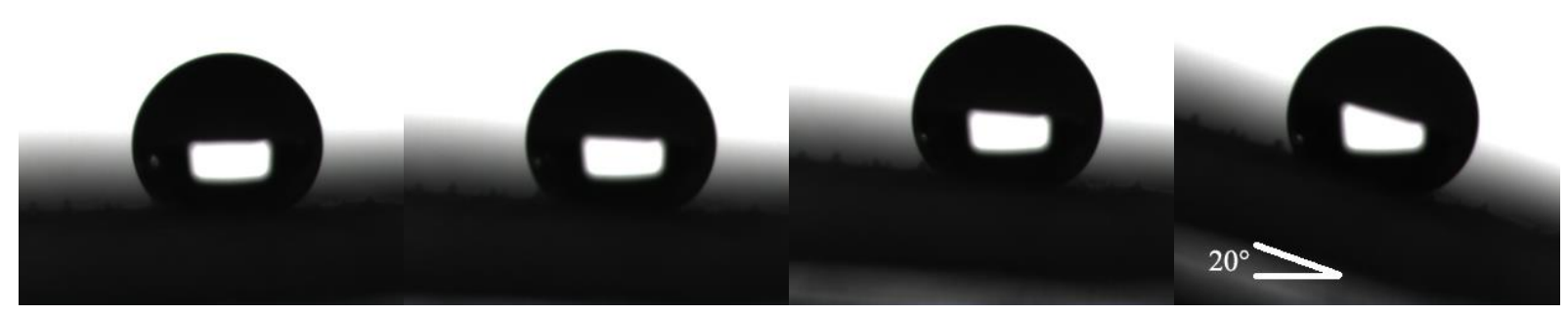

Fig. 10. Contact angle measurements for photopolymer replicas with master leaves of (a) Lotus, (b) Indian shot, (c) bamboo and (d) grape. (e-h) Snap shots of rolling angle tests of water droplet dripped on coated silicon surface covered by photopolymer replica with lotus leaf master. 
The bioinspired coating surfaces are hydrophobic under corrosive conditions. The $\mathrm{pH}$ dependent CAs were depicted in Fig. 11 for the lotus and Indian shot replicas. Clearly, water CAs do not changed much under weak acidic or basic conditions. This is important, because the ambient conditions are accompanied with corrosive liquids such as acid rains, or basic contaminations due to salt migrations (Hautala et al. 2015). Additionally, the polymer coating is advanced in resistance to bases, which easily etch glass substrates or Si itself.

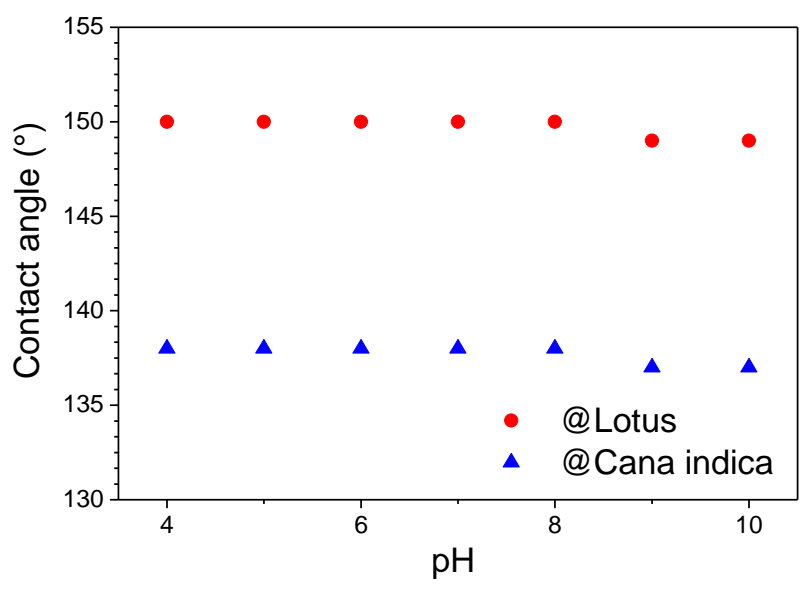

Fig. 11. Variation of water contact angle with the different $\mathrm{pH}$ values of the dipping water on the $\mathrm{Si}$ slabs coated with lotus replica and Cana Indica replica. Typical experimental error within $\pm 0.5^{\circ}$.

As shown in Fig. 12, a case study was carried out by using a white dust physically adhered on the PPR@Indian shot as an example. A typical photo of Indian shot is depicted in Fig. 12(a), where the stem, leaves and flowers are well shown. After dripping a water drop on to the PPR@Indian shot surface and tilting the surface to $20( \pm 0.5)^{\circ}$, the water drop started to roll. As soon as it touched the dust, the dust followed the water droplet until it was fully removed from the surface. The cleanness of the PV surface was thus restored. The hydrophobic surface is very useful for photovoltaics installed at tilted angles to face the sunlight. Dusts can be removed by water (e.g. rain) rolled by the gravity. Consequently, EQE will be decreased on the PV arrays. 


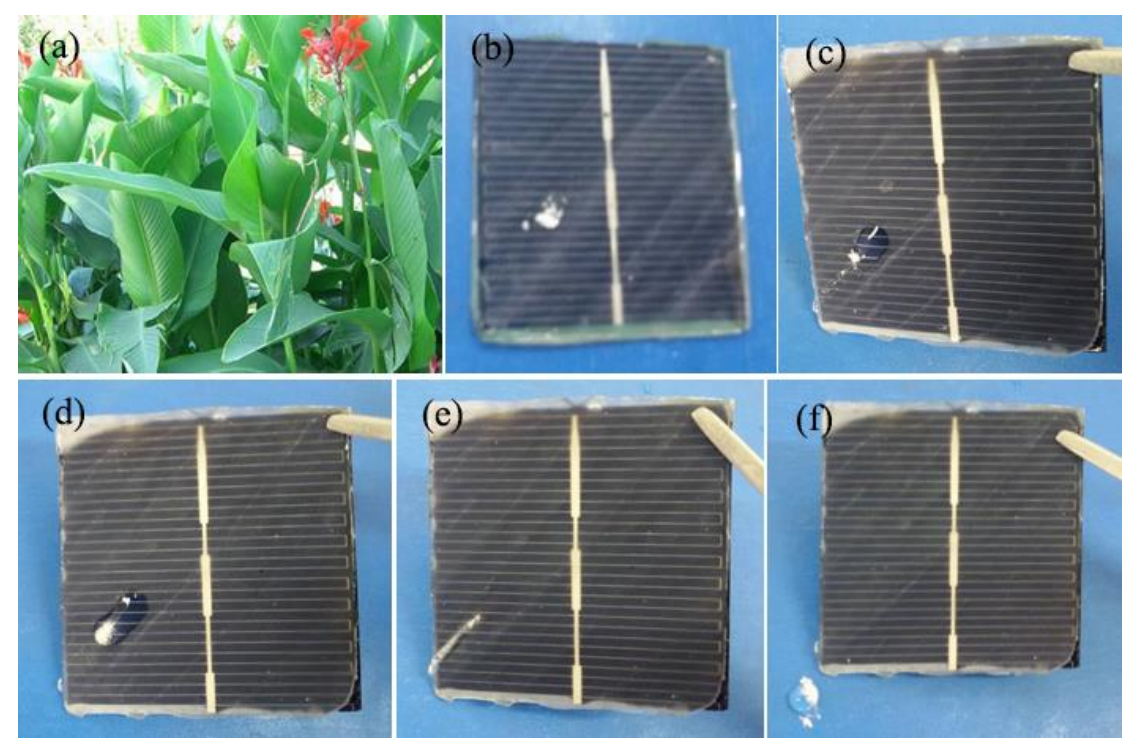

Fig. 12. Photo of Indian shot (a) and dust removal process (b)-(f) on silicon solar cell coated with photopolymer replica with Indian shot as the leaf master.

\section{Concluding remarks}

In conclusion, we have successfully realized a facile route to reach antireflective, hydrophobic, and durable surface on silicon and Si-based photovoltaics via imprinting of leaf surface structures. Thanks to special optical properties of the bio-inspired patterns, the light harvesting efficiency of the solar cells is promoted. A maximum gain of $10.9 \%$ was realized when the lotus leaf was employed as the module master. Benefiting from the unique wettability of the leaves, the PV surface was engineered (super)hydrophobic. Furthermore, large effective areas of the master leaves will also ensure the formation of large coverage area on middle-sized PV cells. We also systematically studied physical mechanisms leading to coating functionalities of the bio-inspired design. Lightwave guidance mechanisms on the bio-coating interfaces were clarified through optical simulations, and wettability through surface state analysis.

Despite these achievements, it is noticed that the polymer coverages are still not antiscratching to hard objects. To print the leaf-mimicking structures onto glass will help to enhance the hardness of the coverage, while using solid mould at the casting stage ensure industrial scale 
fabrication of these structures onto various substrates. Though not perfect yet, this route is hoped to be served as a starting point in promotion of the bionic AR coatings that eventually applied on photovoltaic industry, and awarded with cheers of renewable energies.

\section{Acknowledgements}

This work is financially supported by the National Natural Science Foundation of China (Grant No. 51205001), and Anhui Polytechnic University (No. 2016BJRC005). The Strategic Grant of Oulu University, and the European Union Regional Development Foundation and Oulu Council. The work is also partially supported by Provincial Natural Science Foundation of Hunan, China (2015JJ2138). We also thank Norland Products Inc. for providing optic information of the NOA 63, and Center of Microscopy and Nanotechnology of Oulu University for SEM measurements.

\section{References}

Hirst, Louise C, and N. J. Ekins-Daukes., 2011. Fundamental losses in solar cells. Progress in Photovolt. Res. \& Appl., 19, 286-293.

Said, Syed A. M., N. Al-Aqeeli, and H. M. Walwil., 2015. The potential of using textured and antireflective coated glasses in minimizing dust fouling. Solar Energy, 113, 295-302.

Dev, A., Dev Choudhury, B., Abedin, A., \& Anand, S., 2014. Antireflection: Fabrication of Periodic Nanostructure Assemblies by Interfacial Energy Driven Colloidal Lithography. Adv. Funct. Mater., 24, 4561-4561.

Adamian, Z. N., Hakhoyan, A. P., Aroutiounian, V. M., Barseghian, R. S., \& Touryan, K., 2000. Investigations of solar cells with porous silicon as antireflection layer. Proceed. SPIE-Internat. Society Optic. Engin., 64, 347-351.

Bien, D. C., H. W. Lee, and S. A. Badaruddin., 2012. Formation of silicon nanostructures with a combination of spacer technology and deep reactive ion etching. Nanoscale Res. Lett., 7, 288. 
Zang, Z., Nakamura, A., and Temmyo J., 2013. Single cuprous oxide films synthesized by radical oxidation at low temperature for PV application. Opt. Express, 21, 11448-11456.

Bichotte, M., Kämpfe, T., Iff, W., Celle, F., Reynaud, S., Pouit, T., \& Jourlin, Y., 2017. High efficiency concentrated solar power plant receivers using periodic microstructured absorbing layers. Solar Energy Mater. Solar Cells, 160, 328-334.

Jovanov, V., Moulin, E., Haug, F. J., Tamang, A., Bali, S. I., Ballif, C., \& Knipp, D., 2017. From randomly self-textured substrates to highly efficient thin film solar cells: Influence of geometric interface engineering on light trapping, plasmonic losses and charge extraction. Solar Energy Mater. Solar Cells, 160, 141-148.

Huang, Z., Yang, S., Zhang, H., Zhang, M., \& Cao, W., 2015. Replication of leaf surface structures for light harvesting. Sci. Rep., 5.

Huang, Z., Shi, T., Zhang, H., Zhang, M., Huttula, M., \& Cao, W., 2016. A computational study of antireflection structures bio-mimicked from leaf surface morphologies. Solar Energy, 131, 131-137.

Chen, Y. C., Huang, Z. S., \& Yang, H., 2015. Cicada-wing-inspired self-cleaning antireflection coatings on polymer substrates. ACS Appl. Mater. \& Interfaces, 7, 25495-25505.

Vüllers, F., Gomard, G., Preinfalk, J. B., Klampaftis, E., Worgull, M., Richards, B., Hölscher, H. and Kavalenka, M. N., 2016. Bioinspired Superhydrophobic Highly Transmissive Films for Optical Applications. Small, 12, 6144-6152.

Sun, M., Luo, C., Xu, L., Ji, H., Ouyang, Q., \& Yu, D., et al., 2005. Artificial lotus leaf by nanocasting. Langmuir, 21, 8978-81.

Sharma, C. S., Mattaparthi, S., 2014. Biomimicked high-aspect-ratio hierarchical superhydrophobic polymer surfaces. Bioinspired Biomim. \& Nanobiomat., 3, 4-9. 
Gajaseni, J., N. Gajaseni., 1999. Ecological rationalities of the traditional homegarden system in the Chao Phraya Basin, Thailand. Agrofor. Syst., 46, 3-23.

Li, X., Deng, S., Fu, H., \& Xie, X., 2014. Synergistic inhibition effects of bamboo leaf extract/major components and iodide ion on the corrosion of steel in $\mathrm{H}_{3} \mathrm{PO}_{4}$, solution. Corros. Sci., 78, 29-42.

Leem, J. W., Choi, M., Dudem, B., \& Yu, J. S., 2016. Hierarchical structured polymers for lightabsorption enhancement of silicon-based solar power systems. RSC Adv., 6, 55159-55166.

Huang, Z., Cai, C., Wang, G., Zhang, H., Huttula, M., \& Cao, W., 2016. Structural Color Model Based On Surface Morphology Of Morpho Butterfly Wing Scale. Surf. Rev. and Lett., 23, 1650046.

Koch, K., Barthlott, W., 2009. Superhydrophobic and superhydrophilic plant surfaces: an inspiration for biomimetic materials. Philosophical transactions-Royal Society. Mathematical, Phys. Engin. Sci., 367, 1487-1509.

Choi, D. Y., Oh, Y. S., Han, D., Yoo, S., Sung, H. J., \& Kim, S. S., 2015. Highly Conductive, Bendable, Embedded Ag Nanoparticle Wire Arrays Via Convective Self-Assembly: Hybridization into Ag Nanowire Transparent Conductors. Adv. Funct. Mater., 25, 3888-3898.

Shi, E., Li, H., Yang, L., Zhang, L., Li, Z., \& Li, P., et al., 2013. Colloidal antireflection coating improves graphene-silicon solar cells. Nano Lett., 13, 1776-1781.

Zhu, L., Luo, J. K., Shao, G., \& Milne, W. I., 2013. On optical reflection at heterojunction interface of thin film solar cells. Solar Energy Mat. \& Solar Cells, 111, 141-145.

Nowak, S. H., Banaś, D., Błchucki, W., Cao, W., Dousse, J. C., Hönicke, P., et al., 2014. Grazing angle X-ray fluorescence from periodic structures on silicon and silica surfaces. Spectrochimica Act. B: Atom. Spectr., 98, 65-75. 
Williams, R., \& Goodman, A. M., 1974. Wetting of thin layers of $\mathrm{SiO}_{2}$ by water. Appl. Phys. Lett., 25, 531-532.

Sun, M., Luo, C., Xu, L., Ji, H., Ouyang, Q., Yu, D., \& Chen, Y., 2005. Artificial lotus leaf by nanocasting. Langmuir, 21, 8978-8981.

Guan, H., Han, Z., Cao, H., Niu, S., Qian, Z., Ye, J., \& Ren, L., 2015. Characterization of multiscale morphology and superhydrophobicity of water bamboo leaves and biomimetic polydimethylsiloxane (PDMS) replicas. Journal of Bionic Engineering, 12, 624-633.

Zhu, S.; Yang, X.; Li, T,; Li, F.; \& Cao, W. Phase and morphology controllable synthesis of superhydrophobic $\mathrm{Sb}_{2} \mathrm{O}_{3}$ via a solvothermal method. J. Alloy Comp., in press.

Meng, W., Feng, Z., Li, F., Li, T., \& Cao, W., 2016. Porous coordination polymer coatings fabricated from $\mathrm{Cu}_{3}(\mathrm{BTC})_{2} \cdot 3 \mathrm{H}_{2} \mathrm{O}$ with excellent superhydrophobic and superoleophilic properties. New Journal of Chemistry, 40, 10554-10559.

Zhu, S., Yang, X., Li, T., Li, F., \& Cao, W., 2017. Phase and morphology controllable synthesis of superhydrophobic $\mathrm{Sb}_{2} \mathrm{O}_{3}$ via a solvothermal method. J. Alloys Compd., 721, 149e156.

Hautala, L., Jänkälä, K., Mikkelä, M. H., Tchaplyguine, M., \& Huttula, M., 2015. Surface site coordination dependent responses resolved in free clusters: applications for neutral sub-nanometer cluster studies. Physical Chemistry Chemical Physics, 17, 7012-7022.

Fig. 1. Schematics of fabrication steps (a) A fresh leaf was taken as a master module (b) The leaf was stuck in the die (c) Polydimethylsiloxane was deposited on the leaf to get negative structures of the master. (d) The flexible negative mold was covered with an anti-sticking layer (e) Viscous 
NOA63 was poured onto the negative mold and Si slab was cover onto NOA63. The NOA63 was then polymerized using Ultraviolet light. (f) After polymerization, the detached NOA63 mimicked the master leaf surface structures on Si slab.

(a)

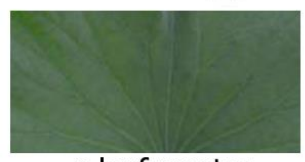

a leaf master

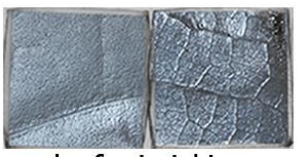

leaf mimicking coating on $\mathrm{Si}$

(f) (b)

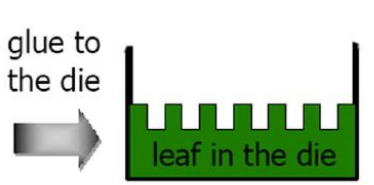

curing
and
relesse

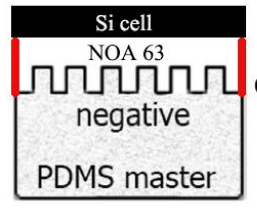

ultraviolet light (c)

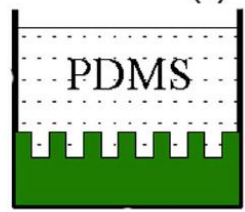

curing

and 西 coating onto $\mathrm{Si}$

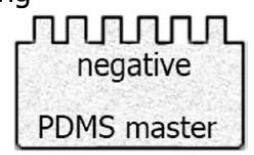

(d)

Fig. 2. Scanning electron microscopic (SEM) determination of surface morphologies of photopolymer replicas compared with these of their master leaves. (a)-(d), SEM images master leaf structures from Lotus, Indian shot, Bamboo, Grape, (e)-(h), from their corresponding replicas, and (i)-(1) the magnified SEM images of the replica.
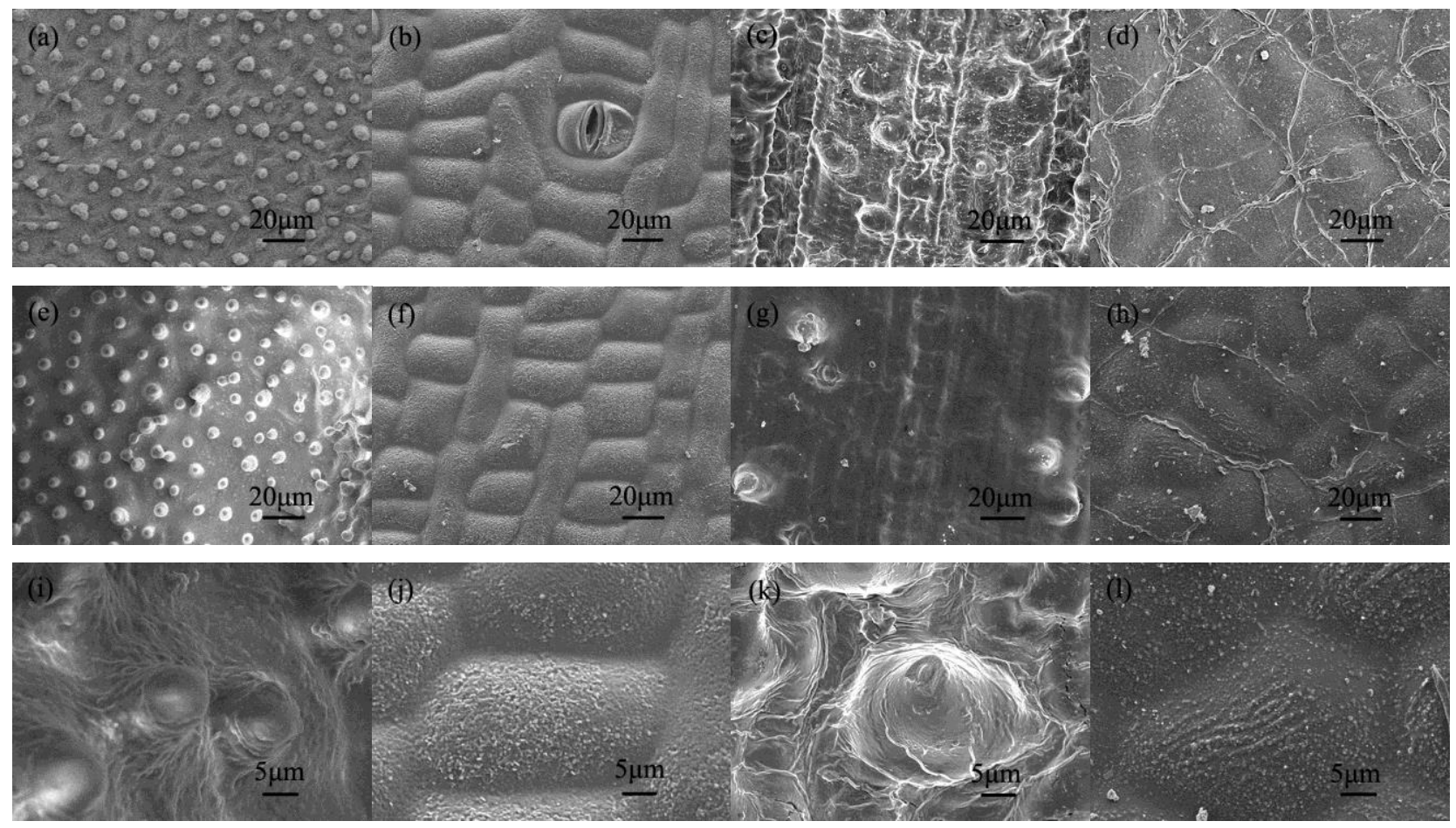
Fig. 3. SEM images of surface morphologies of heating-cooling cycles test samples of mimicked the master leaf surface structures from (a) Lotus (b) Indian shot (c) Bamboo (d) Grape.

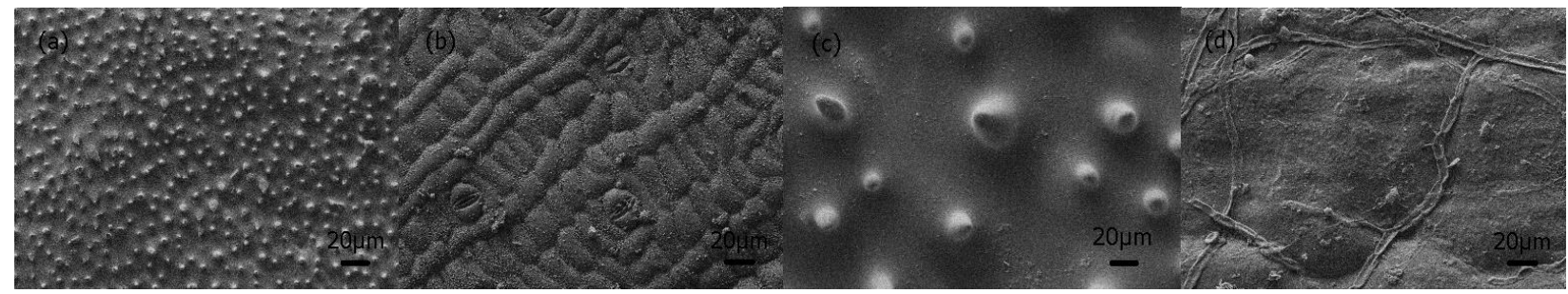

Fig. 4. Cross-sectional scanning electron microscopies of photopolymer-Si interface (a), and individual papillae of the lotus leaf replica curved on flat Si slab (b).
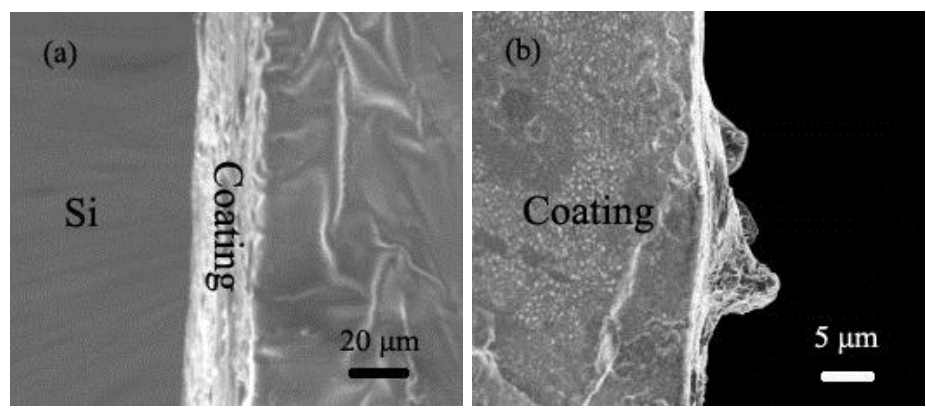

Fig. 5. Photo of polished silicon coated with leaf replicas with their masters of (a) bare polished silicon, (b) PPR@Indian shot, (c) PPR@Lotus, (d) PPR@Grape and (e) PPR@Bamboo from right to left. The very left is a bare polished silicon slab for comparison purpose.

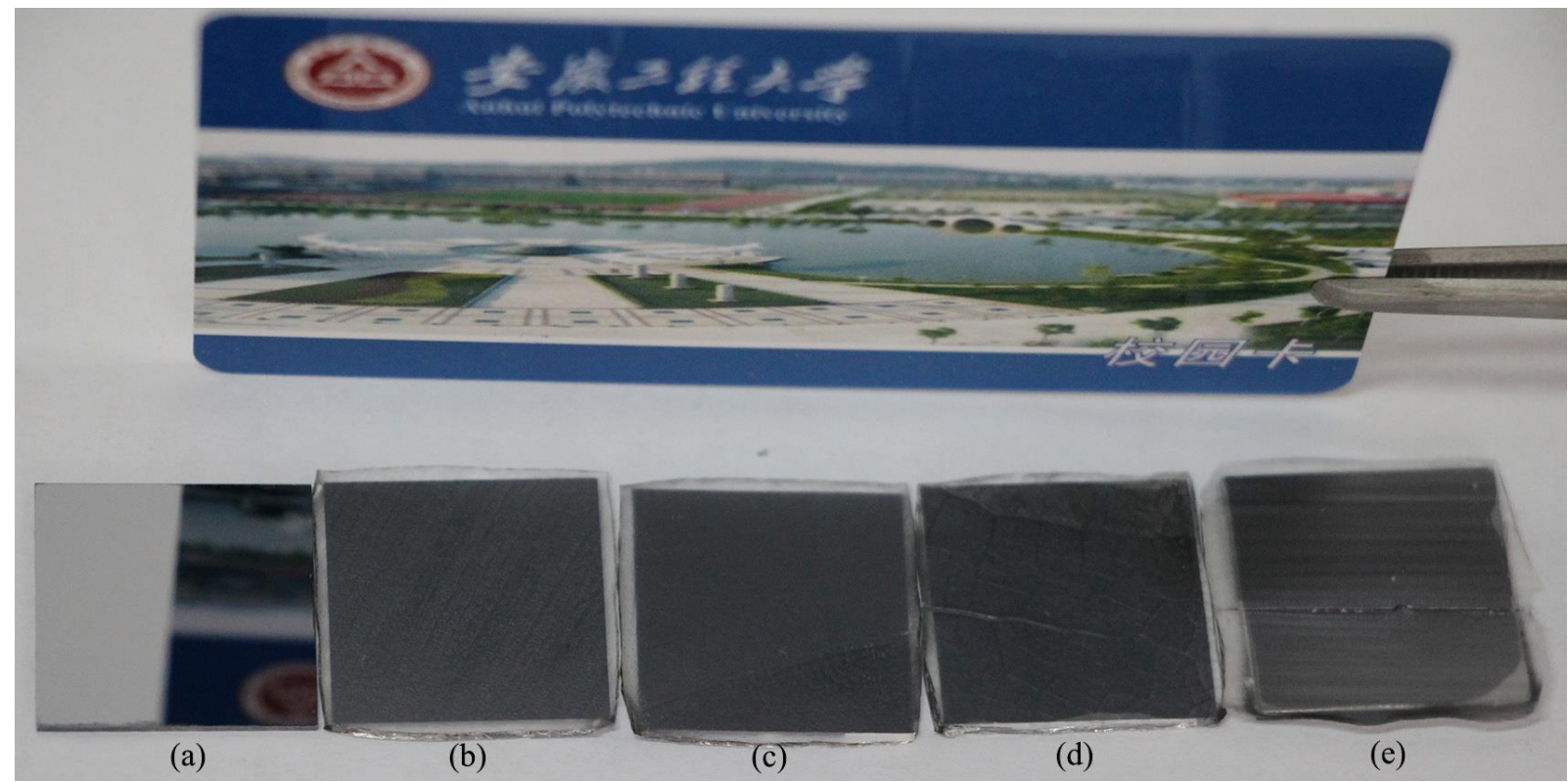


Fig. 6. Reflectance (a) and absorption (b) of plant photopolymer coated silicon surfaces determined through UV-visible spectroscopy.
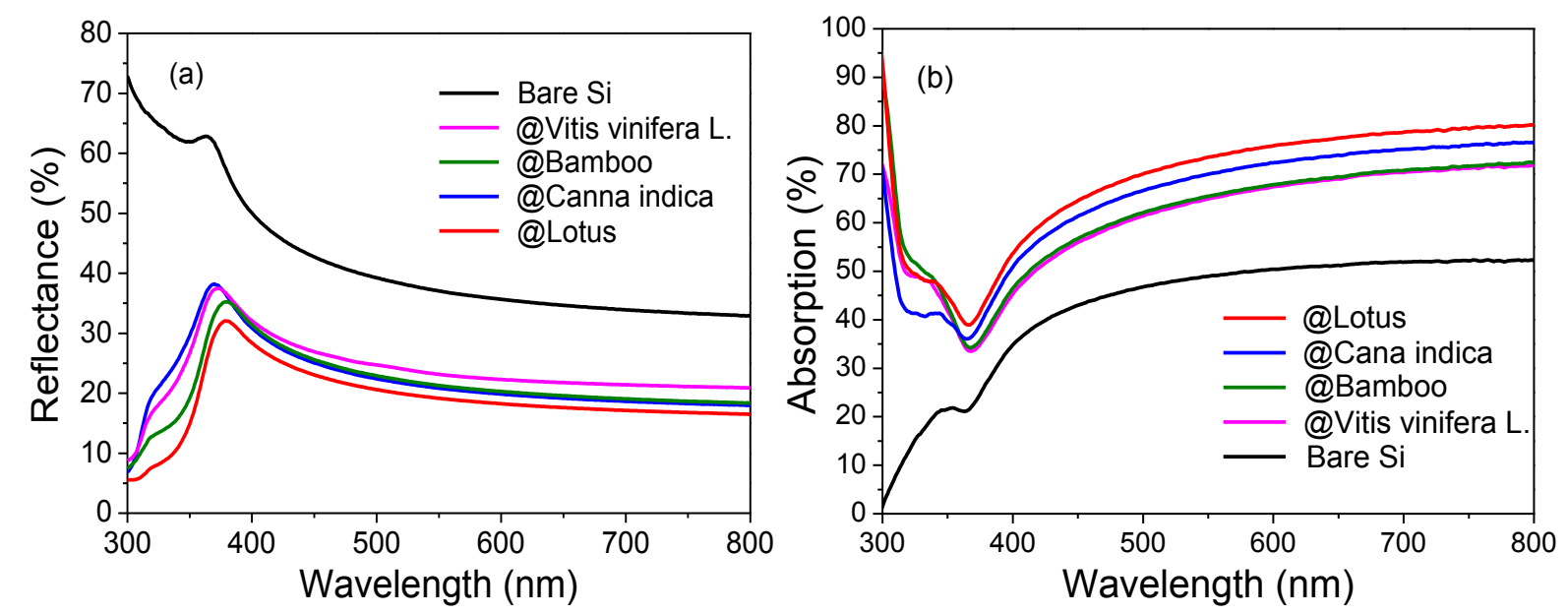

Fig. 7. Micro morphology of abstracted PPR@bamboo model (a) for optical simulation, and calculated reflectance curves from the model, flat $\mathrm{SiO}_{2}$ cover and bare silicon surfaces (b).
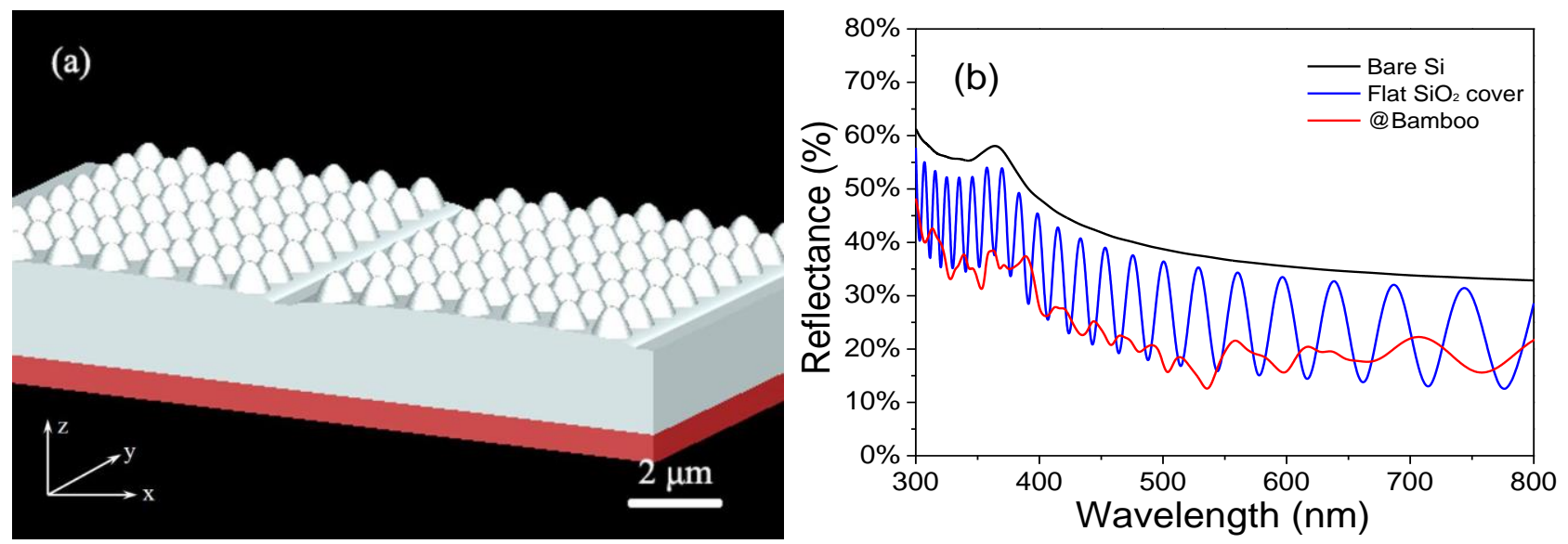

Fig. 8. Light scattering spot. (a) shows the light scattering effect of PPR@Bamboo when a laser with a diameter of $3 \mathrm{~mm}$ passing though the mimicked bamboo leaf structure NOA63 polymer. (b) shows the light scattering effect of smooth NOA63 polymer. 
Fig. 9. Current-voltage curves of the silicon photovoltaics covered by different leaf replicas compared with the cell without any coverage measured under solar simulator. Inset of (a) shows a photo of bare silicon photovoltaics (left) and one covered by Indian shot replica (right), while (b) denotes maximum powers of the photovoltaics.
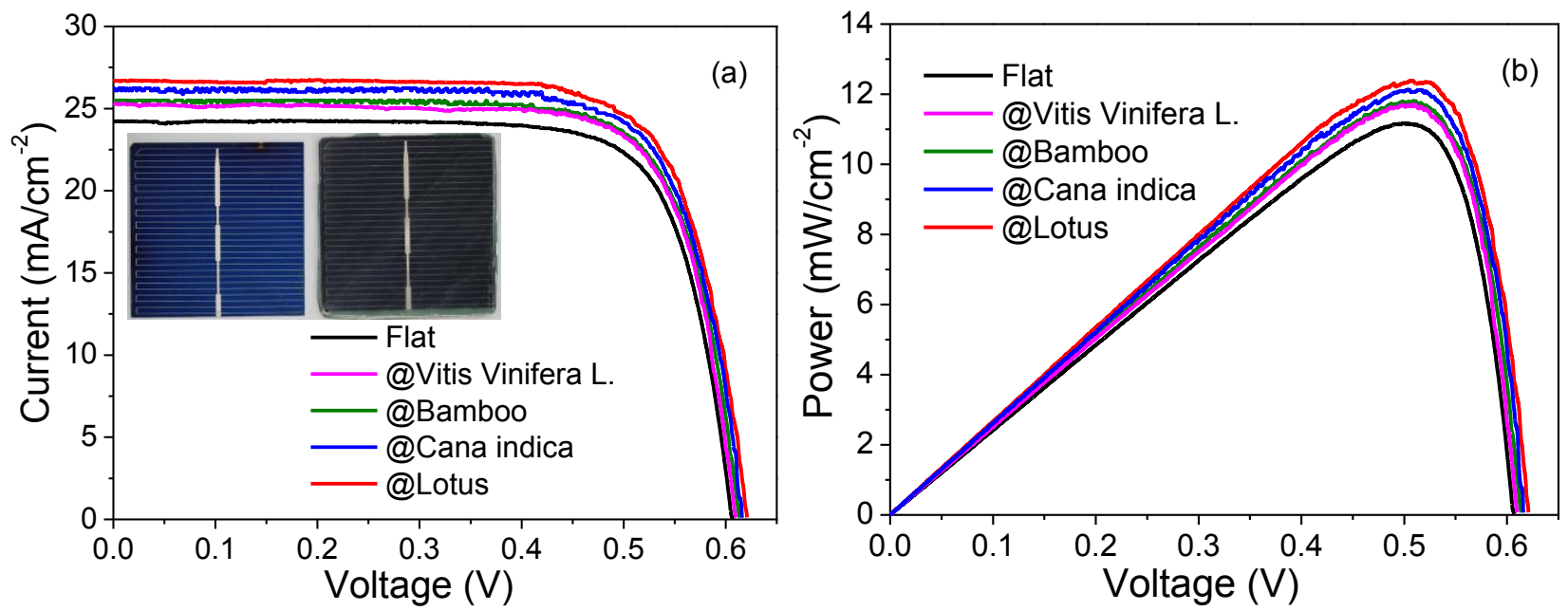

Fig. 10. Contact angle measurements for photopolymer replicas with master leaves of (a) Lotus, (b) Indian shot, (c) bamboo and (d) grape. (e-h) Snap shots of rolling angle tests of water droplet dripped on coated silicon surface covered by photopolymer replica with lotus leaf master. 


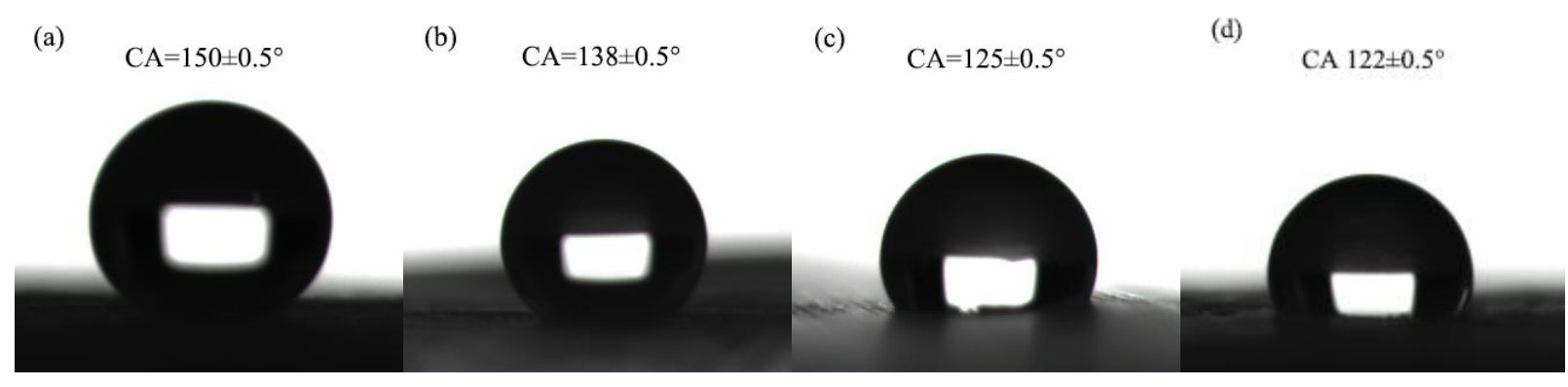

(e)

(f)

(g)

(h)

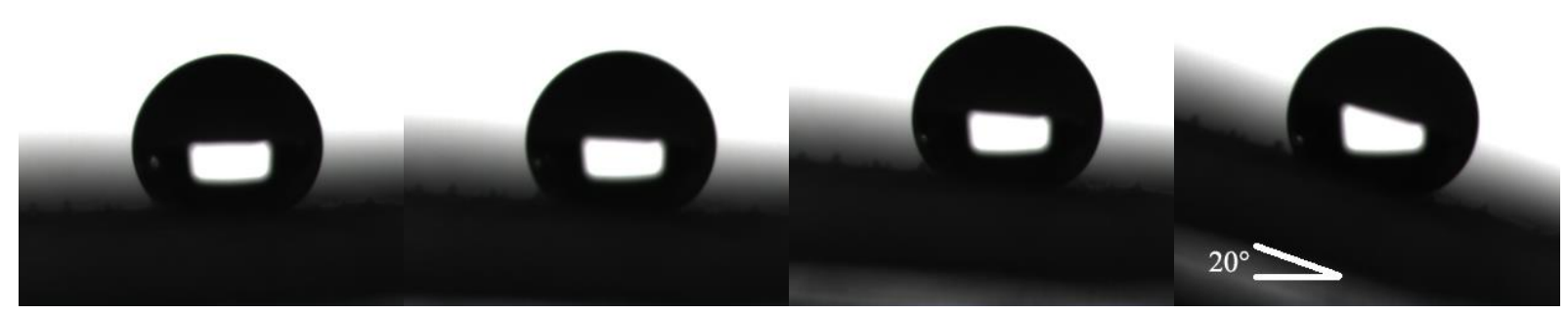

Fig. 11. Variation of water contact angle with the different $\mathrm{pH}$ values of the dipping water on the $\mathrm{Si}$ slabs coated with lotus replica and Cana Indica replica. Typical experimental error within $\pm 0.5^{\circ}$.

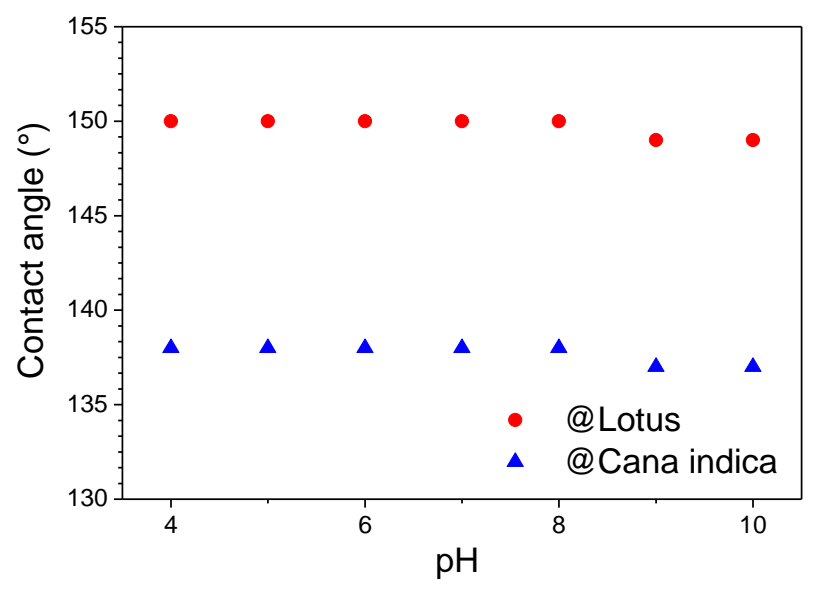

Fig. 12. Photo of Indian shot (a) and dust removal process (b)-(f) on silicon solar cell coated with photopolymer replica with Indian shot as the leaf master. 


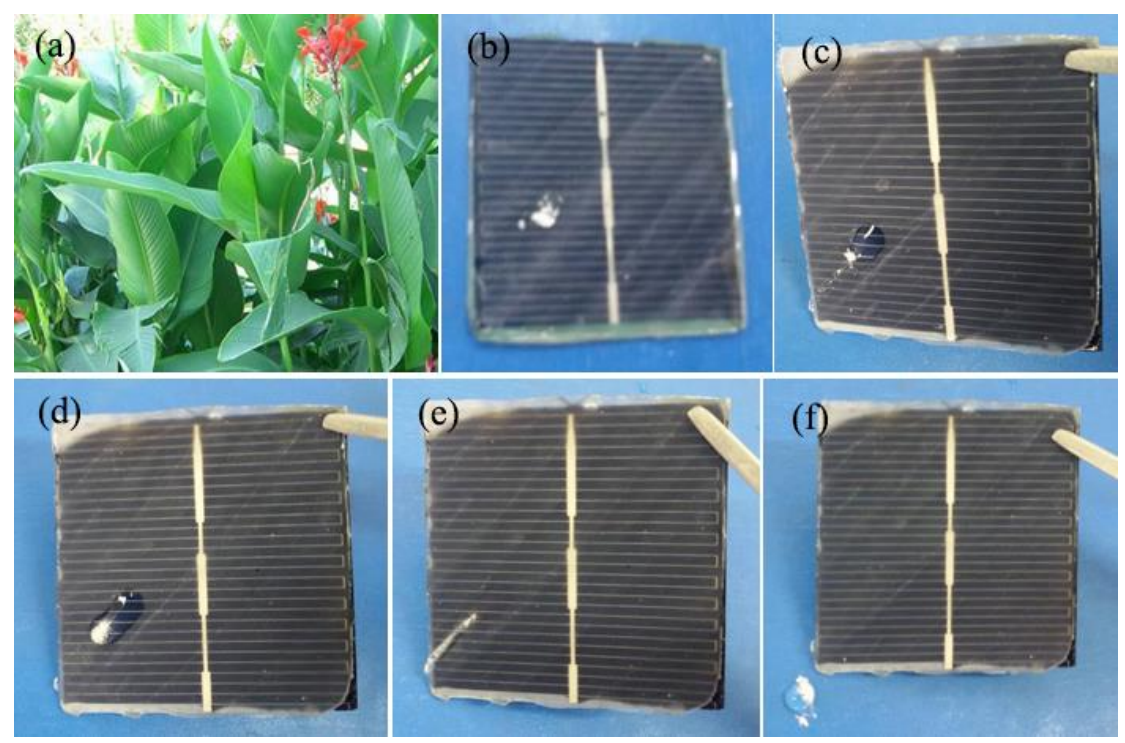

